ARTICLE

\title{
Whole-genome resequencing of 472 Vitis accessions for grapevine diversity and demographic history analyses
}

\author{
Zhenchang Liang ${ }^{1,2}$, Shengchang Duan ${ }^{3,4}$, Jun Sheng 3,5,6, Shusheng Zhu ${ }^{3,6}$, Xuemei Ni ${ }^{7,8}$, Jianhui Shao6, \\ Chonghuai Liu9 ${ }^{9}$ Peter Nick ${ }^{10}$, Fei Du ${ }^{6}$, Peige Fan ${ }^{1}$, Ruzhi Mao ${ }^{6}$, Yifan Zhu ${ }^{3,6}$, Weiping Deng ${ }^{6}$, Min Yang 6 ,

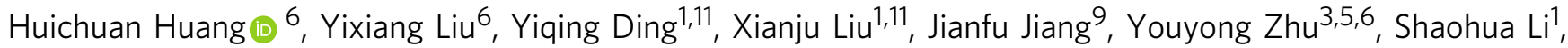 \\ Xiahong $\mathrm{He}^{3,6}$, Wei Chen ${ }^{3,5} \&$ Yang Dong ${ }^{3,5}$
}

Understanding the Vitis species at the genomic level is important for cultivar improvement of grapevine. Here we report whole-genome genetic variation at single-base resolution of 472 Vitis accessions, which cover 48 out of 60 extant Vitis species from a wide geographic distribution. The variation helps to identify a recent dramatic expansion and contraction of effective population size in the domesticated grapevines and that cultivars from the panBlack Sea region have a unique demographic history in comparison to the other domesticated cultivars. We also find selective sweeps for berry edibility and stress resistance improvement. Furthermore, we find associations between candidate genes and important agronomic traits, such as berry shape and aromatic compounds. These results demonstrate resource value of the resequencing data for illuminating the evolutionary biology of Vitis species and providing targets for grapevine genetic improvement.

\footnotetext{
${ }^{1}$ Beijing Key Laboratory of Grape Sciences and Enology, Laboratory of Plant Resources, Institute of Botany, Chinese Academy of Sciences, Beijing 100093, China. ${ }^{2}$ Sino-Africa Joint Research Center, Chinese Academy of Sciences, Wuhan 430074, China. ${ }^{3}$ State Key Laboratory for Conservation and Utilization of Bio-Resources in Yunnan, Yunnan Agricultural University, Kunming 650201, China. ${ }^{4}$ Nowbio Biotechnology Company, Kunming 650201, China. ${ }^{5}$ Yunnan Research Institute for Local Plateau Agriculture and Industry, Kunming 650201, China. ${ }^{6}$ Key Laboratory for Agro-biodiversity and Pest Control of Ministry of Education, Yunnan Agricultural University, Kunming 650201, China. ${ }^{7}$ BGI, BGI-Shenzhen, Shenzhen 518120, China. ${ }^{8}$ BGI Institute of Applied Agriculture, BGI-Shenzhen, Shenzhen 518120, China. ${ }^{9}$ Zhengzhou Fruit Research Institute, Chinese Academy of Agricultural Sciences, Zhengzhou 450009, China. ${ }^{10}$ Botanical Institute, Karlsruhe Institute of Technology, Karlsruhe 76128, Germany. ${ }^{11}$ University of Chinese Academy of Sciences, Beijing 100049 , China. These authors contributed equally: Zhenchang Liang, Shengchang Duan, Jun Sheng, Shusheng Zhu. Correspondence and requests for materials should be addressed to X.H. (email: hexiahong@ynau.edu.cn) or to W.C. (email: wchenntr@gmail.com) or to Y.D. (email: loyalyang@163.com)
} 
D omesticated grapevine (Vitis vinifera ssp. vinifera) is the most cultivated fruit crop in the genus Vitis, which also contains about 60 inter-fertile wild species ${ }^{1-3}$. Within their native habitat in the temperate regions of the world, there are about 28 wild Vitis species indigenous to North America and about 30 wild Vitis species indigenous to East Asia ${ }^{1-3}$. V. vinifera ssp. sylvestris is the only extant wild Vitis taxon native to Europe and Near East, and it is believed to be the wild progenitor for almost 10,000 domesticated grapevine cultivars today ${ }^{1-3}$. In addition, about 1000 commercial grapevine cultivars are interspecific hybrids of the domesticated grapevines and other wild Vitis species ${ }^{2}$. The berries of the grapevine plants are either consumed directly as fresh fruit and raisins or made into various alcoholic and nonalcoholic beverages. In the context of cultural and religious exchange, the agricultural exploitation of grapevines has greatly influenced the human race ever since civilization flourished in the Near East.

Despite the importance of grapevine cultivation in human history and the economic values of cultivar improvement, largescale genomic variation data for grapevines are lacking. Besides the widely used $10-20 \mathrm{~K}$ genotyping arrays ${ }^{4,5}$, the whole-genome resequencing data of various qualities were only reported very recently for 36 grapevine accessions in total ${ }^{6-8}$. The deficit of genomic resource has hampered the investigation of the past history and the present trait improvement of grapevines. For example, current knowledge based on archeological excavation and chemical analysis of the wine potteries in Georgia argued for the earliest viniculture in the Near East at about 8000 years ago 9 . This domestication origin for grapevine was supported by a genotyping analysis of 1000 accessions $^{10}$, and the distribution of chlorotype variations of 1200 grapevines suggested an additional area of origin in the western Mediterranean ${ }^{11}$. However, the domestication time and the demographic history of grapevine based on genomic data remain elusive. Secondly, the biology of grapevine went through significant changes during domestication, with the most noticeable ones being seed shape and flower $\operatorname{sex}^{3}$. Without large-scale variation data, it is impossible to discern genomic regions under selective sweep on a global scale that might give rise to important domestication traits. Lastly, the majority of the domesticated grapevine cultivars are susceptible to disease. At the end of the 19th century, mildews and Phylloxera pests from North America devastated the vineyards and wild grapevines in Europe ${ }^{3}$. For this reason, a sustainable viticulture will rely on harnessing the genetic diversity of not only grapevines in cultivation, but also Vitis species in the wild ${ }^{10}$.

With these points in mind, we propose to delineate the wholegenome genetic variations at single-base resolution from 472 Vitis accessions and four closely-related species in other genera of the Vitaceae family from a wide geographic distribution (Supplementary Data 1). These accessions include 64 wild European accessions ( $V$. vinifera ssp. sylvestris, WEU), 44 accessions from 26 wild East Asian species (WEA), 35 accessions from 21 wild North American species (WNA), 220 accessions from 177 domesticated grapevine cultivars (V. vinifera ssp. vinifera, CEU), and 109 accessions from 69 interspecific-hybrid grapevine cultivars (HYB). Considering that there are about 11,000 acknowledged grapevine cultivars and 60 Vitis species, this resequencing collection as an effort of three institutions aims to capture as many genetic variations in the Vitis species as possible, provide the largest resource to date to facilitate the breeding of new grapevine cultivars, and serve as a stepping stone for more extensive collaborations in future to investigate grapevine genetic diversity. With the identified single-nucleotide polymorphisms (SNPs), we find a recent dramatic expansion and contraction of effective population size in the domesticated grapevines and a unique demographic history in cultivars from the pan-Black Sea region. We also find selective sweeps for berry edibility and stress resistance improvement. Furthermore, we find associations between candidate genes and important agronomic traits, such as berry shape and aromatic compounds.

\section{Results}

SNPs and genomic structural variations. About $4.1 \mathrm{~Tb}$ of wholegenome sequencing data (27.3 billion paired-end raw reads) were generated for 472 Vitis accessions and four other closely-related species at an average depth of $\sim 15.5 \times$ (Supplementary Fig. 1). The mapping rate of these raw reads to the $V$. vinifera reference genome ${ }^{12}$ was $97.4 \pm 4.6 \%$, and the estimated error rate was $0.02 \pm 0.01 \%$ (Supplementary Data 2). Additionally, the genome coverage was more than $80 \%$ across all chromosomes for the majority of accessions (Supplementary Fig. 2).

In order to assess the genetic diversity in both wild and cultivated Vitis accessions, we mapped all individuals to the Pinot Noir reference genome ${ }^{12}$, a method also used in the analyses of large resequencing datasets of other plant genera, such as Malus $^{13}$, Citrus ${ }^{14}$, and Cajanus ${ }^{15}$. After applying basic filtering criteria (see Methods) we identified 77,726,929 SNPs, $10,278,017$ short genomic insertions and deletions (indels), and about 25,000 copy number variants. Further filtering yielded a basic set of 37,859,960 SNPs and 3,854,659 indels ( $\leq 40 \mathrm{bp}$ ) with minor allele frequency (MAF) more than 0.005 , and a core set of $12,549,273$ SNPs and 904,280 indels $(\leq 40 \mathrm{bp}$ ) with MAF more than 0.05 . The ratios of transition to transversion (Ti/Tv) SNPs for the basic set and core set were estimated to be 2.48 and 2.88, respectively, showing the high quality of the SNP call sets.

A survey of all the identified indels and SNPs in the grapevine genome showed that every chromosome contained regions of high indel and SNP density that deviated from the whole genome averages (Fig. 1). About $73.7 \%$ of SNPs were located in the intergenic regions and $4.0 \%$ in the coding sequences. The nonsynonymous-to-synonymous substitution ratio for the SNPs in the coding regions was 1.17 , which is comparable to the values reported for pigeonpea $(1.18)^{15}$, but lower than the values reported for tomato $(1.23)^{16}$, soybean $(1.35)^{17}$, and rice $(1.46)^{18}$. We also found that $73.6 \%$ of indels were located in the intergenic regions and $1.3 \%$ in the coding regions. An estimated $66.9 \%$ of indels in the coding regions could cause frameshift mutations.

Analysis of Vitis phylogeny. The core set of SNPs were used to analyze the phylogeny and population structure of the Vitis accessions. Maximum likelihood (ML) phylogenetic analysis with 100 nonparametric bootstraps revealed distinct monophyletic clades for wild North American Vitis species (WNA, purple), wild East Asian Vitis species (WEA, yellow), wild European species (WEU, red), and domesticated grapevine cultivars (CEU, dark green, Fig. 2a and Supplementary Fig. 3). This topology supports previous reports that the Vitis clade containing wild North American species (New World species) is sister to the Eurasian Vitis clade containing wild East Asian, wild European, and domesticated grapevine species ${ }^{19,20}$. Within the Eurasian Vitis clade, wild European Vitis is a sister clade to domesticated grapevine species, which in turn, as a whole clade, is sister to wild East Asian Vitis clade. A closer examination of the phylogenetic nodes (bootstrap value $\geq 75$ ) showed that at least two and five smaller groups existed in the wild North American and wild East Asian Vitis clades, respectively (Supplementary Fig. 3). The majority of interspecific-hybrid grapevine cultivars (HYB, light green) were classified into two separate clusters (Supplementary Fig. 4), mainly reflecting their various hybridization background among wild North American, wild East Asian, and domesticated grapevine cultivars. The ML phylogenetic tree also revealed a few 

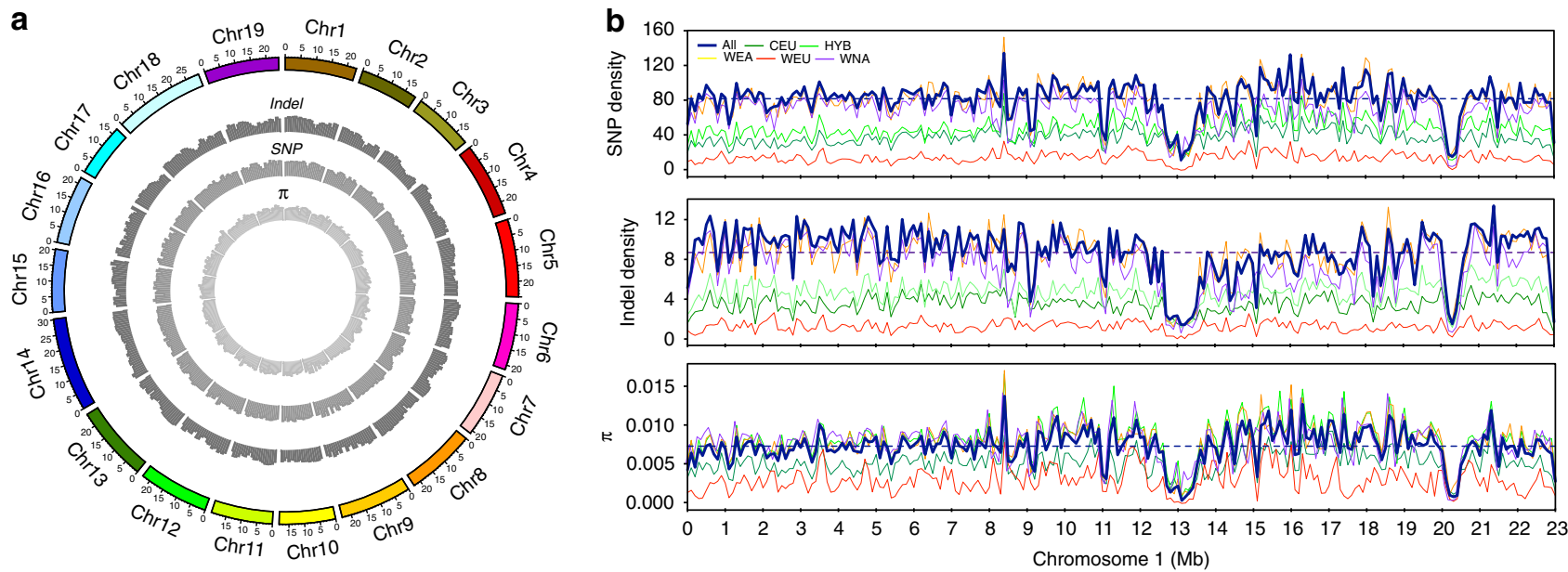

Fig. 1 Summary of the genomic variations identified across 472 Vitis accessions. a Circos graph representation of the SNP density, indel density, and total genetic diversity $(\pi)$ across all chromosomes of the grapevine genome scaled to Mb size. b Panoramas of the SNP density, indel density, and total genetic diversity across chromosome 1 of the grapevine genome with contiguous $100 \mathrm{~kb}$ subregions. The dashed line represents the average values for the entire genome. WNA (purple), WEA (yellow), WEU (red), CEU (dark green), and HYB (light green) represent wild North American Vitis species, wild East Asian Vitis species, wild European grapevine, domesticated grapevine cultivars, and interspecific-hybrid grapevine cultivars, respectively. This color scheme is used throughout the manuscript
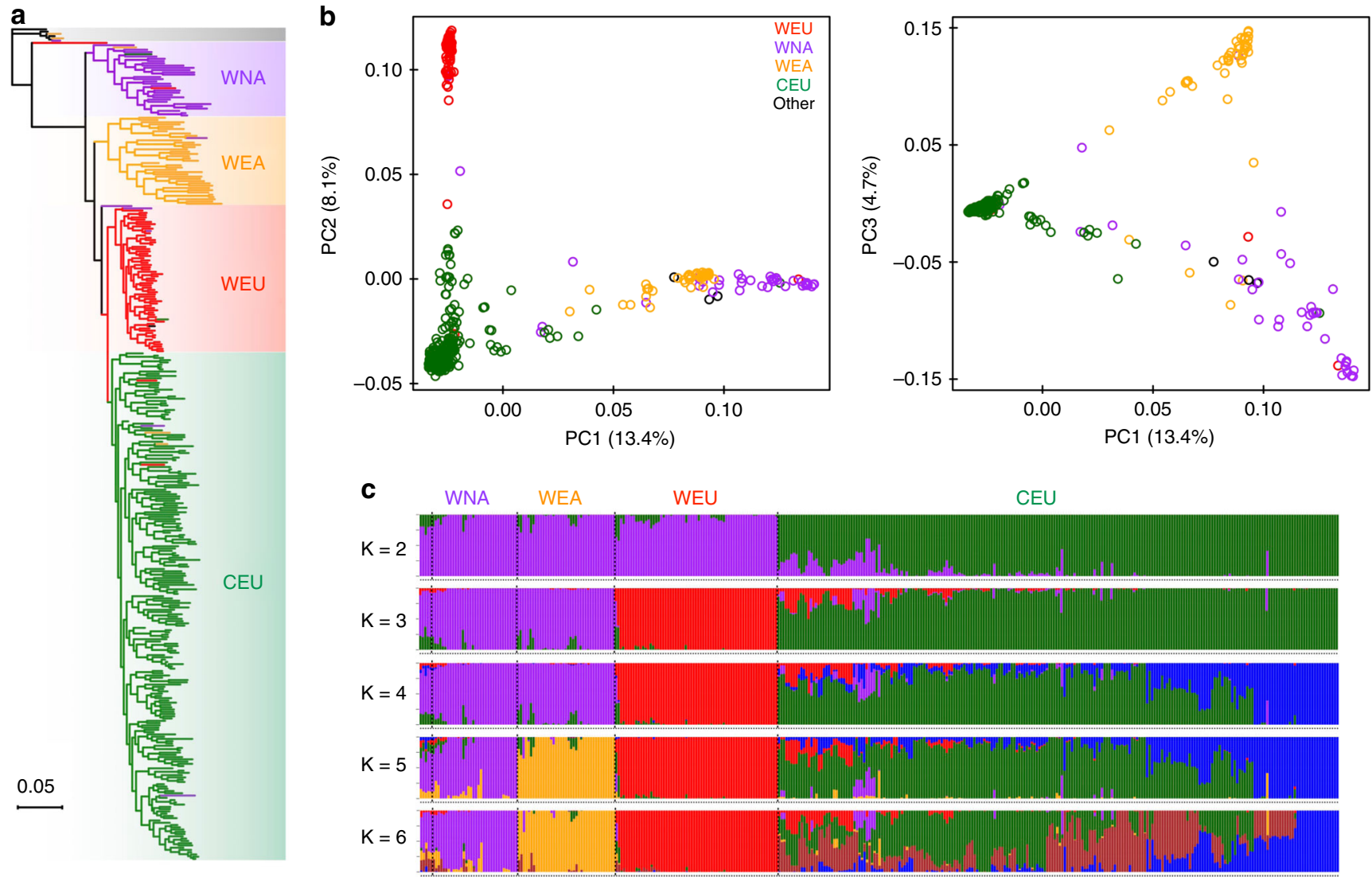

Fig. 2 Phylogeny and population structure of major Vitis categories. a Maximum likelihood phylogenetic tree of grapevine accessions inferred from the whole-genome SNPs with 100 nonparametric bootstraps (see Supplementary Fig. 3 and 4 for bootstrap values as blue circles). Parthenocissus tricuspidata (Boston ivy) was used as an outgroup. b PCA plots of the first three components of major Vitis accessions using whole-genome SNP data. $\mathbf{c}$ Population structure of major Vitis categories estimated by ADMIXTURE. Each color represents one ancestral population. Each accession is represented by a bar, and the length of each colored segment in the bar represents the proportion contributed by that ancestral population 
accessions being grouped into other Vitis clades of different genetic background, suggesting potential misclassification of accessions based on morphology during sample collection ${ }^{21}$. For instance, TA-5901 from St. Andres of Canary Islands was originally believed to be $V$. vinifera. The phylogenetic tree suggested that this tree-like specimen might well be a $V$. sylvestris from Northern Africa. Another example is TA-6147, which was identified as a $V$. sylvestris in the UC Davis collection (introduced to KIT in 2006). A previous study using plastid markers cast doubt on this taxonomy as it clustered with wild North American species $^{22}$. Our phylogenetic tree confirmed this misidentification, and showed that TA-6147 might be a Vitis acerifolia sample. A complete description of these misidentifications is in Supplementary Data 1.

Analysis of Vitis population structure. Principal component analysis (PCA) showed substantial genetic diversity among major grapevine categories, with the first three principal components explaining $13.4 \%, 8.1 \%$, and $4.7 \%$ of the total genetic variance, respectively (Fig. 2b). PC1 separated Eurasian accessions (WEU and CEU) from wild East Asian and wild North American accessions, suggesting that the latter two grapevine categories shared more similarity in the genetic background than Eurasian accessions. This finding is further supported by the result of model-based analyses of population admixture (Fig. 2 c, $K=3$; and Supplementary Fig. 5a, c). PC2 evidently separated wild European accessions from domesticated grapevine accessions, whereas PC3 set wild North American accessions apart from wild East Asian accessions (Fig. 2b). The differentiation between these major grapevine categories was also evident in the population admixture graph (Fig. 2c, $K=5$; and Supplementary Fig. 5a and 5c). Additional PCA analysis showed that the interspecific-hybrid grapevine cultivars were strewn in between other grapevine categories (Supplementary Fig. 5b). Their relative positions in the graph reflected the level of genomic influence from each parent Vitis species. In the admixture plot at $K=4$ (Supplementary Fig. 5c), it is apparent that the majority of interspecific-hybrid grapevine cluster 1 (HYB1) received genetic contributions from wild North American, wild East Asian, and domesticated grapevine species, whereas the interspecific-hybrid grapevine cluster 2 (HYB2) received genetic contributions from only wild North American and domesticated grapevine species. This result is in line with the ML phylogenetic tree (Supplementary Fig. 4). Even though most of the domesticated grapevine accessions were closely clustered in the PCA graphs, they showed a clear pattern of high genetic heterogeneity as evidenced by the population admixture analyses (Fig. 2c, $K=6$; and Supplementary Fig. $5 c, K=8$ ).

Analysis of linkage disequilibrium. Characterization of the linkage disequilibrium ( $\mathrm{LD}$, expressed as $r^{2}$ ) pattern is crucial to forward genetics studies in plant ${ }^{23}$. Previous evaluations of LD decay in wild European and domesticated grapevine cultivars using small-scale genetic markers yielded inconsistent results, with some studies showing a relatively small LD extents at $10-20 \mathrm{~kb}^{10,24}$, whereas the others showing a large LD extents at $28-458 \mathrm{~kb}^{5,25}$. With the whole-genome SNPs available, we found that the decay of LD reached half of maximum average $r^{2}$ at a distance of $2.9 \mathrm{~kb}$ for wild European grapevines and $350 \mathrm{bp}$ for domesticated grapevines (Fig. 3a). These parameters are substantially smaller than previous reports and those found in wild soybean $(\sim 27 \mathrm{~kb})^{17}$, wild rice $(20 \mathrm{~kb})^{26}$, and wild maize $(22 \mathrm{~kb})^{27}$. The relatively slower decay of LD in wild European species versus domesticated grapevine cultivars is concordant with previous findings ${ }^{24}$, but it is important to realize that this difference may narrow with a more diverse wild European Vitis population. In comparison to wild European species, we also found that LD decayed rapidly to an average $r^{2}$ of 0.1 within $300 \mathrm{bp}$ for both wild North American and wild East Asian Vitis species (Fig. 3b).

Demographic histories of WEU and CEU grapevines. The demographic history of the domesticated annual crops, such as maize 28,29 and African rice ${ }^{30,31}$, is characterized by a sequential contraction and expansion of estimated effective population size $\left(N_{e}\right)$ around the time of domestication. However, this pattern (particularly $N_{e}$ expansion) was not observed in the domesticated grapevine, a perennial crop with the capability of vegetative propagation $^{6}$. To revisit this question, we applied the multiple sequentially Markovian coalescent (MSMC) ${ }^{32}$ model to the analysis of phased SNP data from both wild European grapevines and domesticated grapevines (Fig. 4a). The results were scaled to real time by assuming a generation time of 3 years ${ }^{10}$ and a neutral mutation rate of $5.4 \times 10^{-9}$ per year (see Methods). As shown in Fig. 4a, wild European grapevines (red lines) experienced a steady decline of $N_{e}$ from the highest point $\left(N_{e} \approx\right.$ $600,000)$ at $400 \mathrm{Kya}$ to the nadir $\left(N_{e} \approx 20,000\right)$ at $10 \mathrm{Kya}$. Similar demographic patterns were observed for the majority of the domesticated grapevines in the world (green lines), with the lowest $N_{e} \approx 60,000$ around 10-20 Kya. Interestingly, domesticated grapevines from the pan-Black Sea region (maroon lines, Caucasus region and west coast of Black Sea) manifested a unique mild $N_{e}$ expansion $\left(N_{e} \approx 150,000\right.$ up to $\left.N_{e} \approx 300,000\right)$ around 30-70 Kya and a subsequent moderate $N_{e}$ contraction $\left(N_{e} \approx 300,000\right.$ down to $\left.N_{e} \approx 60,000\right)$ around $10-30$ Kya. Figure $4 \mathrm{a}$ also shows that the population of all domesticated grapevines started to differ from wild European grapevines at a similar time around 250-300 Kya. From analyzing eight haplotypes for pairs of populations in the MSMC split analyses (Fig. 4b), we found that the relative cross coalescence rate reached 0.5 , suggesting the predicted split state, at around 250-400 Kya between wild European grapevines and domesticated grapevines (all lines). After population divergence, the change in the relative crosscoalescence rates for the pan-Black Sea cultivars followed two unique patterns (maroon lines): one group resembled the Western European cultivars (dark green line) and the other group resembled the East Asian cultivars (light green line; Fig. 4b).

Since the power of MSMC is quite limited for predictions more recent than $10 \mathrm{Kya}$ in this study, we explored the recent demographic history of grapevines with unphased SNP data using SMC $++^{33}$. Single population approach revealed that domesticated grapevines $(n=218)$ experienced a prominent $N_{e}$ expansion (Fig. $4 \mathrm{c} ; N_{e} \approx 40,000$ up to $N_{e} \approx 3,000,000$ ) around 3000 ya to $40 \mathrm{Kya}$ and a subsequent severe $N_{e}$ contraction (down to $N_{e} \approx 5000$ ) around 400-3000 ya. The wild European grapevines experienced $N_{e}$ expansion and contraction during similar period of time (Fig. 4 c; $N_{e} \approx 15,000$ up to $N_{e} \approx 300,000$, then down to $\left.N_{e} \approx 80,000\right)$. The split analyses using domesticated grapevines of various geographical regions (Fig. 4d) showed that the divergence times of pan-Black Sea, Western European, and East Asian cultivars from wild European grapevines were at about 80-100 Kya. The pan-Black Sea and East Asian cultivars also seemed to diverge from the wild European grapevines earlier than Western European cultivars. The $N_{e}$ expansion started earlier for the pan-Black Sea cultivars $(\sim 30 \mathrm{Kya})$ than for Western European ( $22 \mathrm{Kya})$ and East Asian cultivars ( $18 \mathrm{Kya})$. Due to the small sample size of the available pan-Black Sea cultivars, the ensuing $N_{e}$ contractions for these subgroups of grapevines could not be inferred (Fig. 4d). Taking both MSMC and SMC++ results together, we propose that the pan-Black Sea cultivars underwent a unique demographic history ever since their 

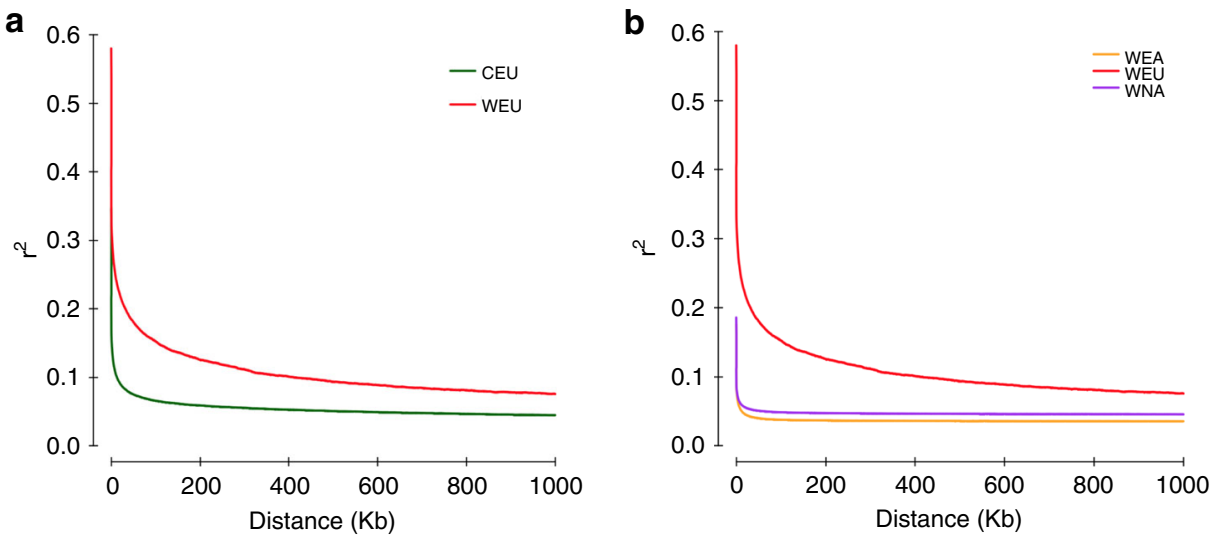

Fig. 3 Decay of linkage disequilibrium of major Vitis categories. a Decay of linkage disequilibrium in grapevine genome for wild European grapevines and domesticated grapevine cultivars. b Decay of linkage disequilibrium in grapevine genome for extant wild Vitis species

a

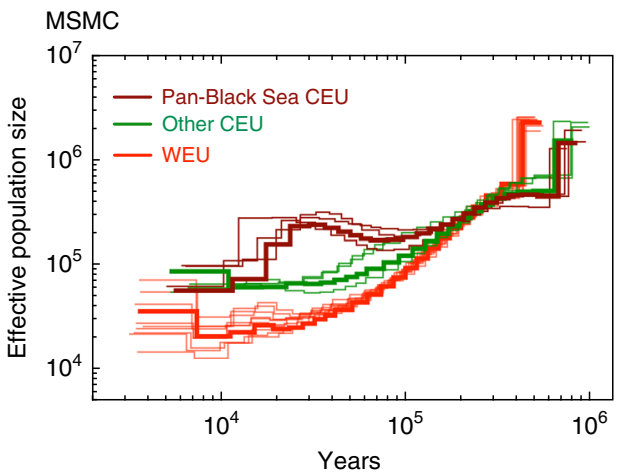

C

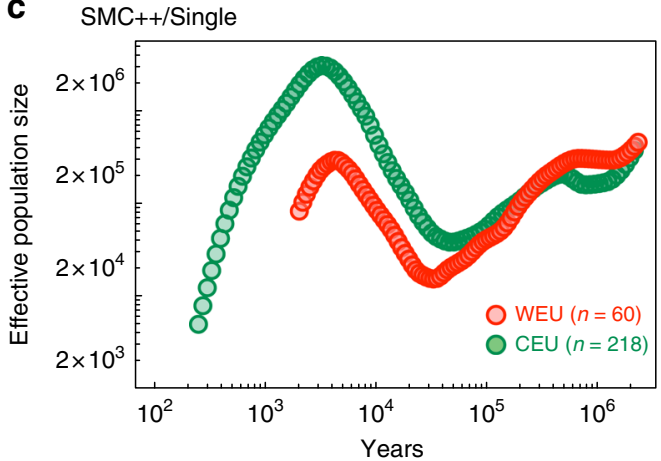

b MSMC-Split

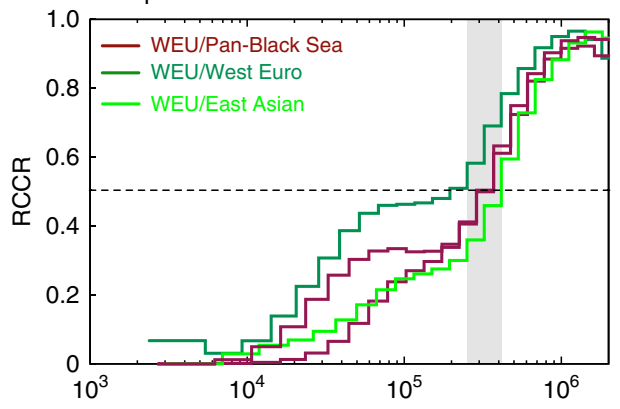

d $\mathrm{SMC}++/$ Split

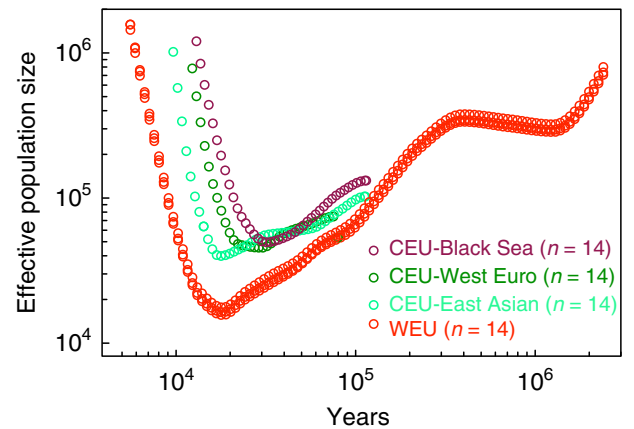

Fig. 4 Demographic history of wild European grapevines and cultivated grapevines. a MSMC-derived demographic history of WEU and CEU grapevines from $10^{4}$ to $10^{6}$ years ago. Each line represents a run of four haplotypes from two individual accessions. An example was highlighted in each group. For CEU grapevines, cultivars from the pan-Black Sea region generated distinct demographic history compared with other cultivars. b MSMC split analysis of WEU and various subgroups of CEU grapevines based on relative cross-coalescence rate (RCCR). c Demographic history of WEU ( $n=60)$ and CEU grapevines $(n=218)$ from $10^{2}$ to $10^{6}$ years ago from SMC++ single population analysis. $\mathbf{d}$ Demographic history of WEU $(n=14)$ and various subgroups of CEU $(n=14)$ grapevines from $10^{4}$ to $10^{6}$ years ago from SMC ++ split analysis

divergence from the wild European grapevines. In addition, all grapevines may have undergone at least one bottleneck since the Last Ice Age (see Discussion). Due to the difficulty in identifying a true progenitor population in grapevine research, we advise all analyses involving the wild European grapevines be interpreted with caution.

Pedigree analysis within Vitis accessions. The accurate reconstruction of grapevine genealogy from genomic data is difficult due to the coexistence of vegetative propagation and sexual reproduction ${ }^{10}$. Nevertheless, we analyzed the patterns of identity-by-descent (IBD) relationships among the 472 Vitis accessions. The histogram of IBD values from pairwise Vitis comparisons is bimodal (Supplementary Fig. 6a). No pairwise IBD values exceeded 0.95 , the empirical cut-off for defining clonality ${ }^{10}$. By defining a cut-off IBD value of 0.420 (lowest value that separates two modes), we found that 335 Vitis accessions (71.0\%) were related to at least one other accession by a firstdegree relationship. When the lowest pairwise IBD value (0.466) for 43 confirmed Vitis parent-offspring pairs was used ${ }^{10}, 292$ Vitis accessions (61.9\%) retained a first-degree relationship with at least one other accession (Supplementary Fig. 6b, c).

The pedigree network via Cytoscape showed that the majority of the first-degree relationships were among accessions in the 


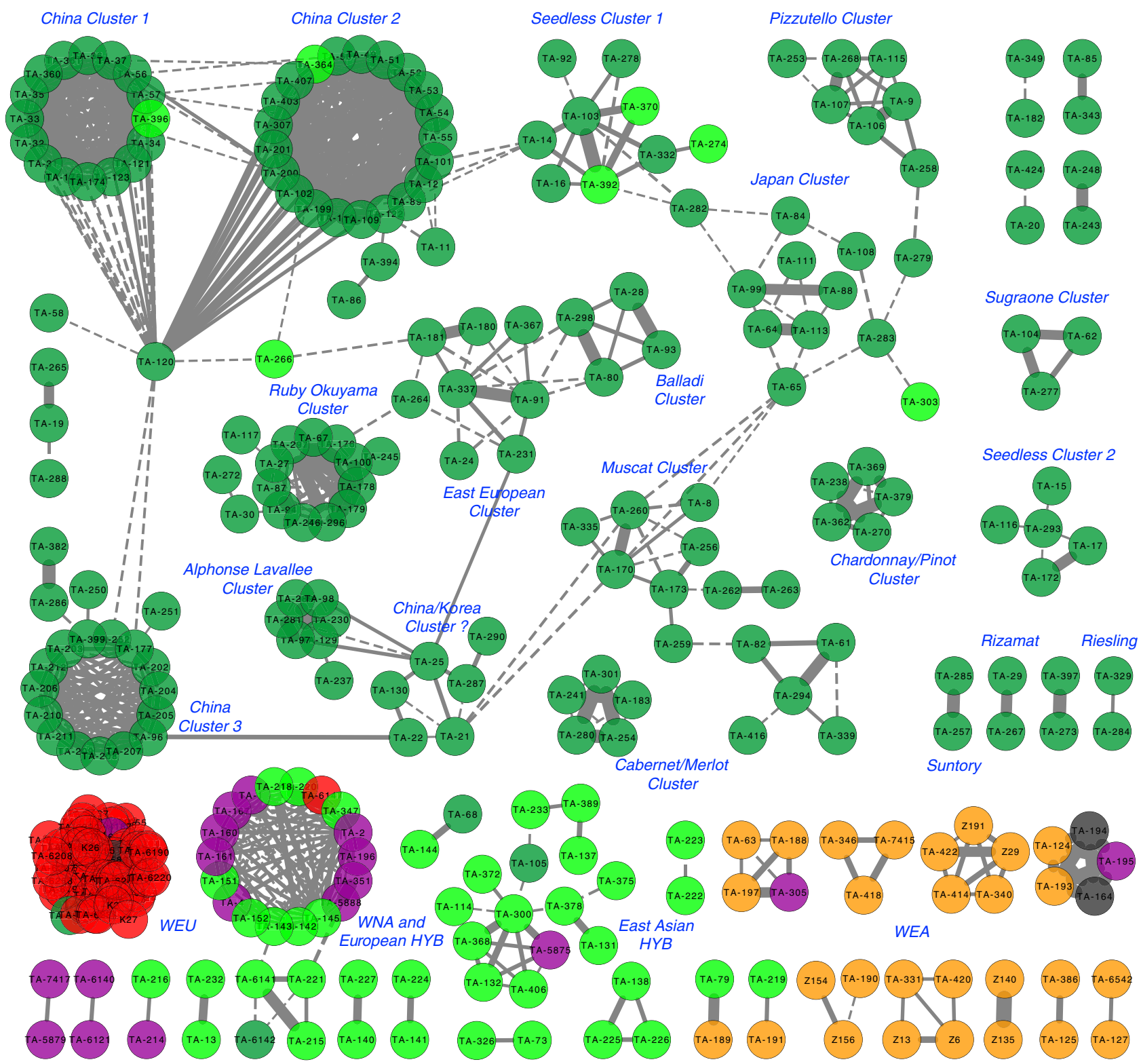

Fig. 5 First-degree relationship network of 335 Vitis accessions. Dashed line represents an IBD value in between 0.420 and 0.466 . Solid line represents an IBD value equal to or greater than 0.466 . The thickness of the line is proportional to the calculated IBD value. Source data is provided as a Source Data file

same major Vitis categories (Fig. 5). The wild European grapevines formed a compact stand-alone cluster. In contrast, the wild North American species were highly connected to the European interspecific hybrid cultivars, agreeing with the history that wild North American grapevines were extensively used in hybridization to counter mildews and pests in the 19th century European vineyards ${ }^{34}$. The wild East Asian species formed a loosely-connected cluster, which implies that wild East Asian species have yet to be fully explored for creating hybrid cultivars. The domesticated grapevine cultivars formed 16 discrete clusters. The Chinese cultivars formed three highly-connected compact clusters that were linked by the key node cultivar "Jingxiu" (Fig. 5). In comparison, the European cultivars formed looselyconnected dispersed clusters. For example, independent clusters showed first-degree relationships of "Cabernet Sauvignon/ Merlot" and "Chardonnay/Pinot", which corresponded to a previous report ${ }^{10}$. Another example is the formation of two separate seedless grapevine clusters, with one centered on "Thompson Seedless" and the other on "Emperor". The network of the domesticated grapevine cultivars is an indication of a highly complicated breeding history of European domesticated grapevines that started thousands of years ago.

Selection signals in WEU and CEU grapevines. Across the Vitis genome, the global nucleotide diversity $(\pi)$ was well correlated to the global SNP and indel density (Fig. 1b). Subsequent analysis showed that the degree of polymorphism in the wild European grapevines $\left(\pi=3.50 \times 10^{-3} ; \quad\right.$ S.D. $\left.=1.84 \times 10^{-3}\right)$ was lower than that of the domesticated cultivars $\left(\pi=5.49 \times 10^{-3}\right.$; S.D. $=$ $\left.1.91 \times 10^{-3}\right)$. This deficit in genetic diversity in wild European grapevines versus domesticated cultivars was well noted in several independent Vitis studies ${ }^{1,24,35}$. Given that the wild European grapevines have a notable reduced $N_{e}$ (Fig. 4), their reduced level of diversity may be the result of fragmented native habitat and increased inbreeding in the recent past ${ }^{24,35}$.

Despite this drawback, we investigated potential selective signals in the genomes of wild European and domesticated grapevines 

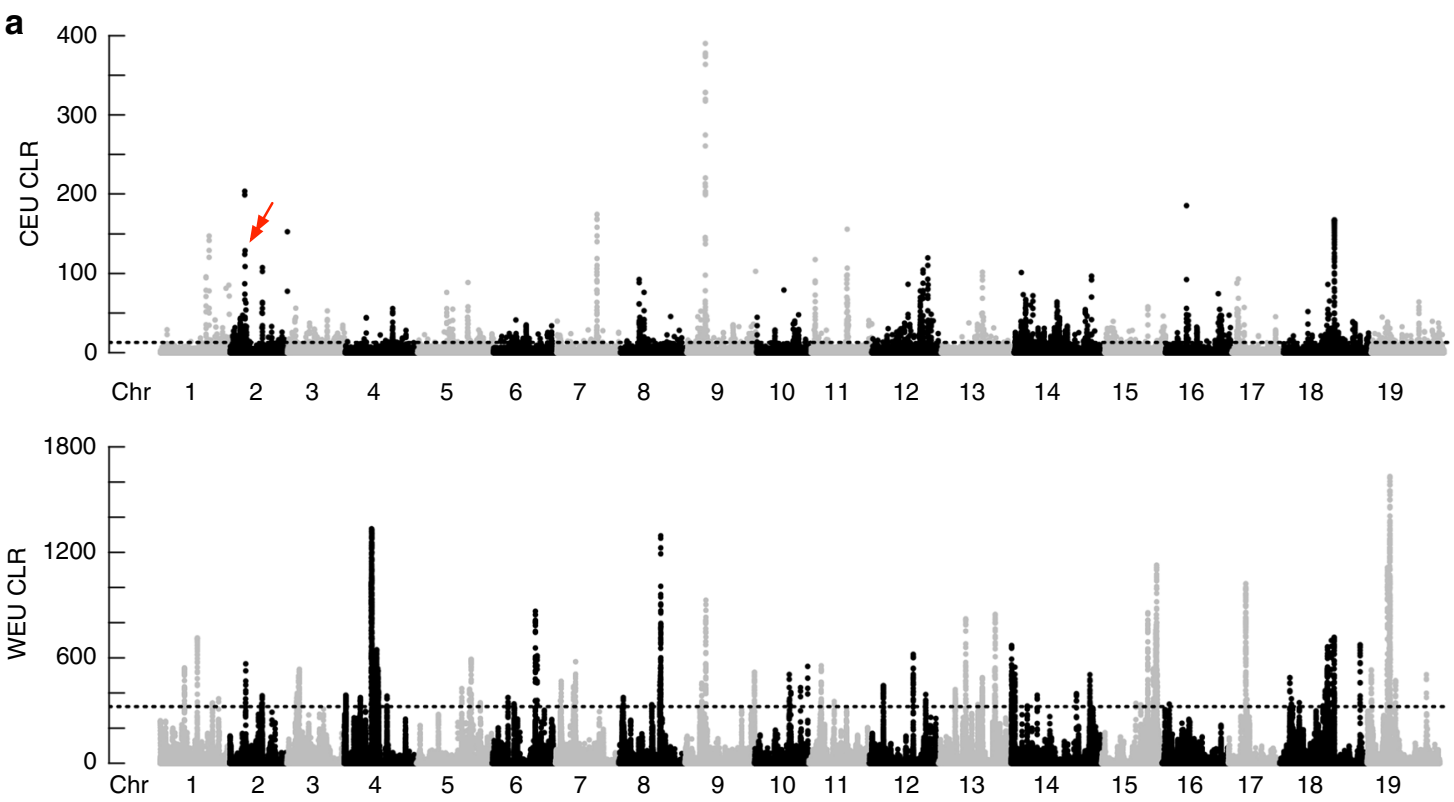

b
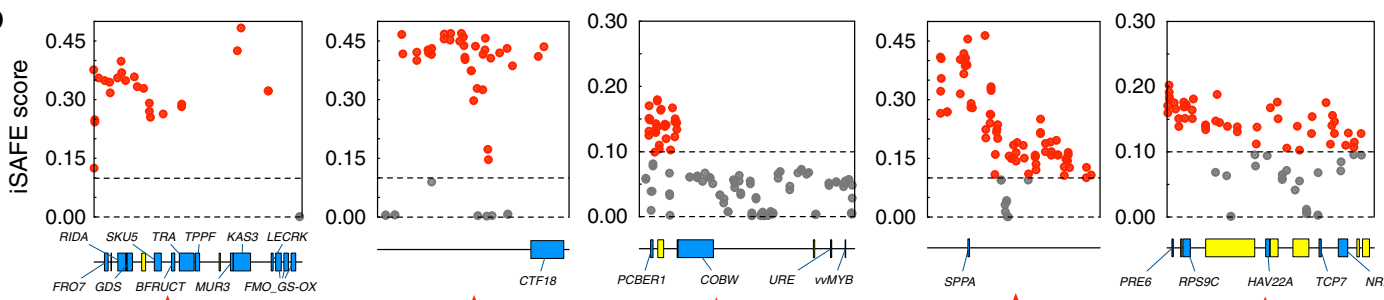

$$
\text { SPPA }
$$
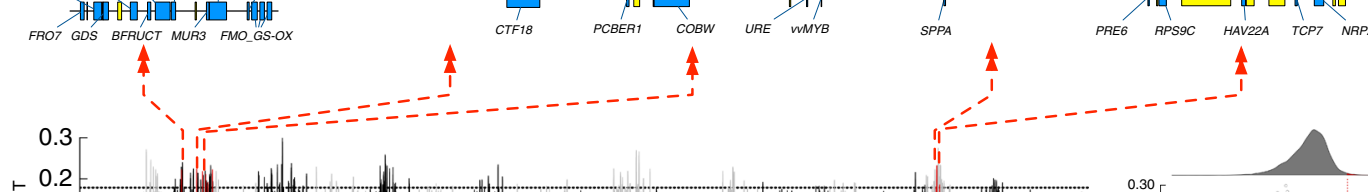

एᄂ

0.0 L
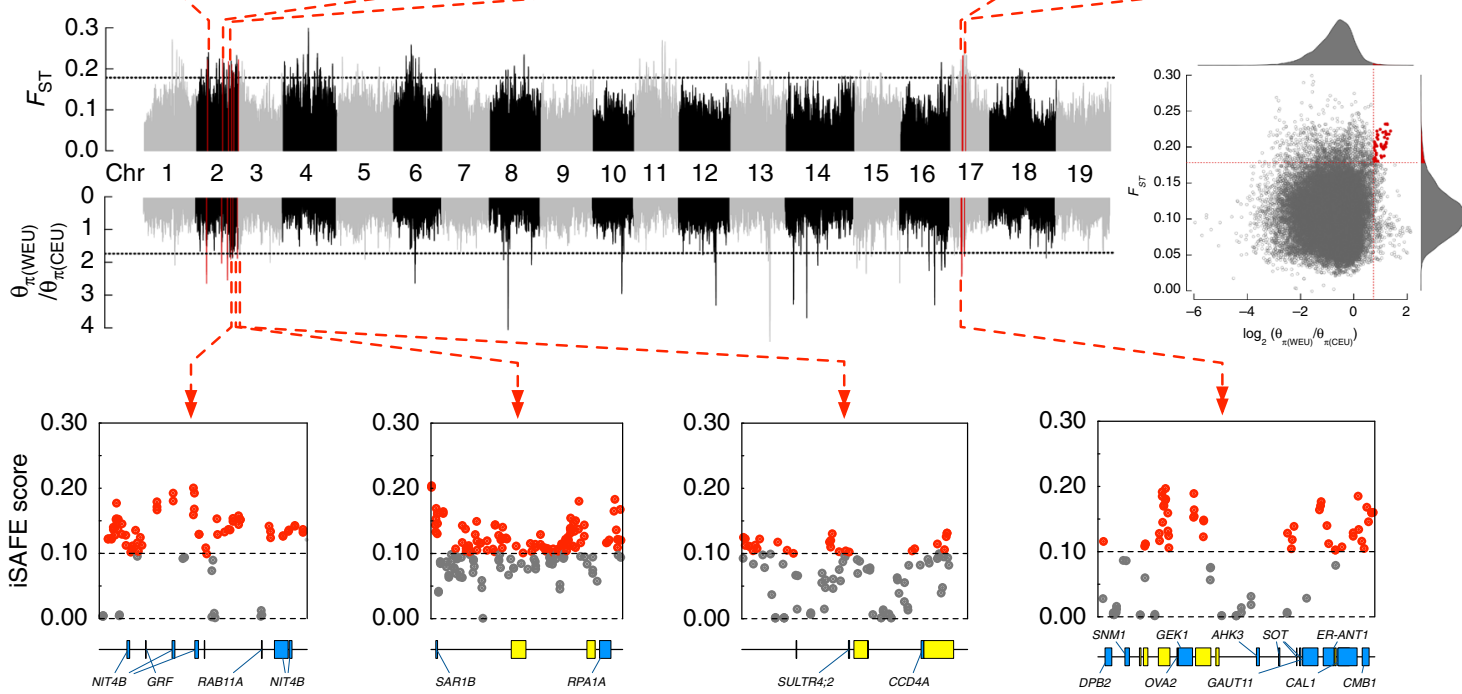

Fig. 6 Selective sweep regions in the grapevine genome. a CLR scores calculated by SweeD across the genome in both wild European and domesticated grapevines. The dashed lines mark the regions at the top $0.5 \%$. The red arrow indicates the putative sex determination region. $\mathbf{b}$ Selective sweep regions during domestication inferred from $F_{\mathrm{ST}}$ and $\pi$ statistics and SNP hotspots in each region influenced by ongoing selection sweep. Each selective sweep region is represented by a red perpendicular line in the genome. Magnified view of each selective sweep region is represented by a horizontal line, on which annotated (blue blocks) and unknown (yellow blocks) genes are drawn. Each dot in the iSAFE plots represents the average iSAFE score for 36 SNPs (step size 18 SNPs) in the region. The dashed line shows the empirical significance cut-off. In the first iSAFE plot, this region overlaps with the putative sex determination region

by identifying the regions (about $1 \mathrm{~kb}$ in length) that scored top $0.5 \%$ in the CLR analysis (Fig. 6a). The 2119 selective sweep regions in the domesticated grapevine genome harbor 1016 candidate genes, whereas the 2120 selective sweep regions in the wild grapevine genome contain 348 candidate genes (Supplementary
Data 3). The numbers of identified genes were larger than those found in a previous study (1016 versus 308 with 28 in common; 348 versus 88 without common genes $)^{6}$. In particular, the previously identified flower sex determination locus on chromosome $2\left(4.91-5.05 \mathrm{Mb}^{36}\right.$ or $\left.4.88-5.04 \mathrm{Mb}^{37}\right)$ is found to contain 
15 significant CLR signals in the domesticated grapevine genome. No overlapping selective sweep regions were found in the genomes of wild European and domesticated grapevines and only 18 genes were found in both lists (Supplementary Data 3). GO enrichment of the 1016 domesticated grapevine candidate genes showed significant functional representation in the GO categories of immune response, regulation of cell death, fructose metabolic process, plasma membrane, and vesicle trafficking (Supplementary Fig. 7). Interestingly, jasmonates-induced cell death was found to be associated with abiotic defense responses in grapevine ${ }^{38}$. Also, the vesicle trafficking of anthocyanins plays important roles in the berry skin coloration and berry ripening in grapevines ${ }^{39,40}$. This result suggests that domesticated grapevines may have been mainly selected for higher fructose content, various ripening times, and broader cultivation areas. In comparison, GO enrichment of 348 wild European grapevine candidate genes showed significant functional representation in the GO categories of macromolecule metabolism, aromatic compound biosynthesis, and jasmonic acid metabolic pathways, suggesting a natural selection for resistance to biotic and abiotic stresses (Supplementary Fig. 7).

Even though our wild European grapevines may not fully reflect the genetic diversity of the true progenitor wild European grapevine population, we decided to identify some potential selective signals during grapevine domestication (wild European versus domesticated cultivars) by surveying the genomic regions that showed high nucleotide diversity differences and $F_{\mathrm{ST}}$ values (both top 5\%). A total of nine domestication-selective sweep regions on two chromosomes were detected, containing 73 genes (Fig. 6b, Supplementary Data 4). One of the selective sweep regions on chromosome $2(4.83-4.97 \mathrm{Mb})$ overlaps with the previously identified flower sex determination locus ${ }^{36,37}$, agreeing with the aforementioned result of CLR analysis (Fig. 6b, first iSAFE plot). We then identified the SNP hotspots favored by ongoing selective sweep (SNP window size $=36$, allele frequency $0.1<v<0.9$ ) within the identified regions using iSAFE analysis (iSAFE $\geq 0.1$, empirical $\left.P<1 \times 10^{4}\right)^{41}$. The result showed that the majority of the SNP hotspots were found in the promoter side of the genes in the selective sweep regions (Fig. $6 \mathrm{~b}$, red dots). The functional annotation of these 73 genes revealed their putative roles in regulating cell growth and metabolism. For instance, a putative $v v M Y B$ gene was identified in a selective sweep region on Chr. 2 with no SNP hotspots around it. This gene has been implicated in the differences of grape skin color ${ }^{42,43}$, possibly through its impact on anthocyanin synthesis. A cluster of the bifunctional NITRILASE/NITRILE HYDRATASE (NIT4B) genes was also identified in a Chr. 2 selective sweep region. This gene, fairly conservative among different plant species, is involved in the detoxification of cyanide and the recovery of nitrogen from cyanogenic glycosides, which affects both plant development and plant defense ${ }^{44}$. Moreover, the TRANSALDOLASE (GANAB) gene and the ACID BETA-FRUCTOFURANOSIDASE (BFRUCT) gene on Chr. 2 suggest that carbohydrate metabolism is a key target of domestication-selective sweep.

Genome-wide association analyses. Previous studies using 1020k SNP arrays have successfully identified grapevine alleles that are associated with important phenotypes, such as seedlessness, muscat aroma, skin color, and flower sex ${ }^{5,43}$. Here, we performed genome-wide association analysis (GWAS), using a compressed MLM model, on the data of 24 grapevine phenotypes that were obtained over 1-3 years (Supplementary Data 5). We detected SNP signals for berry shape, number of seeds, panicle type, berry sucrose content, berry acid content, and 12 aromatic compounds, but not for berry weight, Brix score, and most of berry sugar contents (Supplementary Fig. 8-12 Supplementary Data 6,
Supplementary Data 7). Among all these grapevine traits, berry shape was the only one that had been associated with SNP signals over multiple years. Specifically, five genomic loci containing nine genes (12 SNPs) were found to be associated with berry shape for the years 2016, 2017, and the pooled mean data. One exonic SNP (Chr7.20085154, C to T) causes a nonsynonymous mutation in a putative SERINE/THREONINE-PROTEIN KINASE $(S R K 2 A)$ gene. Even though its physiological function in grapevine is unclear, members of the serine/threonine kinase family are regarded as the central units linking hormonal and environmental stimuli to changes in metabolism and gene expression ${ }^{45}$. These data suggest that it requires even larger sample sizes of grapevines to consistently discern trait-associated SNPs.

\section{Discussion}

In this study, we reported the characterization of genome-wide SNPs from 472 Vitis accessions, an endeavor that covered 48 out of 60 extant Vitis species. The genomic variation data of this scale, though the largest ever reported for grapevine, are in no comparison to the genetic diversity of over 11,000 domesticated grapevine cultivars and wild species around the world. Preferably, this study shall act as the foundation for future more extensive collaborations on a par with the 3,000 Rice Genomes Project ${ }^{46}$. With this said, we were able to utilize our SNP dataset to revisit and provide information about the genetic diversity and demographic history of the domesticated grapevines.

In an ideal scenario, a true wild progenitor population is required for the delineation of grapevine domestication history and the identification of grapevine selective sweep regions in the genome. Inclusion of grapevine samples from such a population in the actual studies, however, has been proved to be difficult ${ }^{6,10}$. For one reason, the morphological similarity between wild European and domesticated grapevines makes the identification work prone to error ${ }^{3}$. Another reason is the inter-fertile nature of Vitis species (gene flow between two subspecies) and the possibility of feralization of the domesticated grapevines ${ }^{3,35}$. Moreover, the population of wild European grapevines only exist in fragmented refugia in Eurasia (e.g., Caucasus, Iberian Peninsula, Balkan Peninsula, and so on $)^{47}$, and these subpopulations manifest substantial genetic diversity among them ${ }^{10,35,47}$.

In our study, the majority of the wild European samples came from the Ketsch peninsula on upper Rhine in Germany, which represented one of the largest wild European grapevine populations in Western Europe ${ }^{48}$. Depending on which microsatellite marker was used for analyses, the Ketsch population was genetically closer to either the Iberian population in the west or the Caucasian population in the east ${ }^{48}$. This result suggested that the Ketsch population might be a genetic sink for the wild European grapevines during the Ice Age ${ }^{48}$. Indeed, data from the phylogenetic tree and population structure analyses (Fig. 2) based on whole-genome SNPs strongly supported the majority of Ketsch population as the true wild European grapevines. Interestingly, two Ketsch accessions (KE06 and TA-6264) were found to be possible feral escapees, as they showed ambiguous phylogenetic positions and clustered with domesticated grapevine cultivars. Additionally, the previously reported wild European grapevines from Zhou et al. ${ }^{6}$ also clustered with our domesticated grapevines and feral escapees (Supplementary Fig. 4 and 5). Even though the Ketsch population represents a true sylvestris population and shares genetic diversity with the Caucasian sylvestris accessions $^{48}$, we still caution against equating this Ketsch population with the true wild progenitor population.

Even though our whole-genome SNP data were not able to display the whole scheme of grapevine domestication throughout history, they were able to reveal some interesting details with 
regard to the demographic history of grapevines. Firstly, the divergence time between our wild European and domesticated grapevines was estimated to be around 200-400 Kya (Fig. 4), a time point that greatly predated a previous estimate $(22 \mathrm{Kya})^{6}$ and the proposed grapevine domestication time $(8000 \mathrm{ya})^{9}$. No matter which subpopulation of the domesticated grapevines was tested, this divergence time did not significantly change (Fig. 4). One possible explanation of the result is that the Ketsch population represents an early diverged subpopulation of wild European grapevines. The ancient progenitor population may have split from the Ketsch population (or vice versa) since 200$400 \mathrm{Kya}$, and later gave rise to all of today's domesticated grapevines. However, the question remains whether such an ancient progenitor population survived hundreds of thousands of years till today. If it did survive, what would be the relationship between this ancient progenitor population and the wild European accessions in the Caucasus region? Secondly, the $N_{e}$ of both wild European and domesticated grapevines experienced a continuous contraction since the Last Ice Age and a significant expansion since 20-40 Kya (Fig. 4c, d). Interestingly, the time of the $N_{e}$ expansion corresponded to the end of the Last Ice Age ${ }^{49}$. A second significant $N_{e}$ contraction occurred since $3000-4500$ ya for both wild European and domesticated grapevines, and the time of the $N_{e}$ contraction corresponded to a much-debated global drought that decimated ancient civilizations ${ }^{50}$. Unfortunately, there are no methods to determine if the $N_{e}$ expanded once more in the recent 200 years. Given the magnitude and pace of $N_{e}$ change according to our data, we would argue all grapevine populations experienced at least one severe bottleneck. Since the pattern of $N_{e}$ change is similar between the wild European and domesticated grapevines, the bottleneck was more likely driven by global climate change, instead of human activity. This is in line with the previous conclusion that domestication-associated bottleneck ( $N_{e}$ change due to human activity) is weak ${ }^{6,10}$. In addition, the start of $N_{e}$ expansion for the domesticated grapevines was earlier than that for the wild European grapevines (Fig. 4c, d). This probably reflects the early human management of the plant as a food source, lending support for a protracted domestication of grapevine starting a lot earlier than $8000 \mathrm{ya}^{6,10}$. With these said, we would like to point out that more data, especially from the demographic histories of various subpopulations of wild and cultivated Vitis accessions, are needed to confirm global climate change as the main driver of $N_{e}$ change. Thirdly, domesticated grapevines from the pan-Black Sea area had a distinct demographic history compared to their counterparts in other regions (Fig. 4). This is probably due to the continuous introgression of local wild European grapevines into the first domesticated grapevines as they spread across the continent. The introgression process has been widely reported in the previous studies $5,10,11,35$.

Analyses of these SNPs also shed light on a set of domestication selective sweeps that promoted grape berry edibility and aroma signatures. In addition, GWA analysis identified candidate SNP signals that are associated with an arrangement of grapevine traits. However, this approach may require data from more samples to evaluate phenotypes (e.g., berry weight, sugar content) that are highly vulnerable to environmental changes. In conclusion, this large-scale SNP resource of Vitis species will facilitate the breeding of new grapevine cultivars and the investigations into various aspects of grapevine biology.

\section{Methods}

DNA sample preparation and sequencing. 347 Vitis accessions were obtained from the Vitis germplasm repository at the Institute of Botany of Chinese Academy of Sciences in Beijing, 40 accessions from the Zhengzhou Fruits Research Institute of Chinese Academy of Agricultural Sciences in Zhengzhou, and 89 accessions from the Botanical Institute of Karlsruhe Institute of Technology in
Karlsruhe (Supplementary Data 1). All plants were subjected to standard management practice that included cultivation, irrigation, fertilization, pruning, and disease control. Young leaves were collected from the plants and snap frozen in liquid nitrogen. Total DNA was extracted with the DNAsecure plant kit (Tiangen, Beijing). $2 \mu \mathrm{g}$ genomic DNA from each accession was used to construct a sequencing library following the manufacturer's instructions using NEBNext Ultra DNA Library Prep Kit (NEB, USA). Paired-end sequencing libraries with an insert size of approximately $400 \mathrm{bp}$ were sequenced on an Illumina HiSeq 4000 sequencer at Novogene-Beijing. Paired-end resequencing reads were filtered using NGSQCToolkit v2.3.3 $3^{51}$. This step removed reads containing adapter or poly-N, and low-quality reads (reads with $>30 \%$ bases having Phred quality $\leq 25$ ) from the raw data, yielding clean data for subsequent downstream analyses. $5 \mathrm{bp}$ off the $5^{\prime}$ and $3^{\prime}$ end of a read was also trimmed.

Variation calling and annotation. Paired-end resequencing reads were mapped to the $V$. vinifera reference genome (Ensembl Plants Release-31) ${ }^{12}$ with BWA (Version: 0.7.10-r789) ${ }^{52}$ using the default parameters. SAMtools (Version: 1.3.1) ${ }^{53}$ software was used to convert mapping results into the BAM format and filter the unmapped and non-unique reads. Duplicated reads were filtered with the Picard package (picard.sourceforge.net, Version: 2.1.1). After BWA alignment, the reads around indels were realigned, realignment was performed with Genome Analysis Toolkit (GATK, version 3.3-0-g37228af) 54 in two steps. The firs step used the RealignerTargetCreator package to identify regions where realignment was needed, and the second step used IndelRealigner to realign the regions found in the first step, which produced a realigned BAM file for each accession. We also downloaded the reads file for 23 grapevine accessions reported in Zhou et al. ${ }^{6}$ from NCBI under BioProject ID: PRJNA388292, and processed the data with same pipeline.

The variation detection followed the best practice workflow recommended by GATK $^{54}$. In brief, the variants were called for each accession by the GATK HaplotypeCaller ${ }^{54}$. A joint genotyping step for comprehensive variations union was performed on the gVCF files. In the filtering step, the SNP filter expression was set as QD $<5.0\|\mathrm{MQ}<40.0\| \mathrm{FS}>60.0\|\mathrm{SOR}>3.0\|$ MQRankSum $<-10.0 \|$ ReadPosRankSum $<-8.0 \| \mathrm{QUAL}<30$, and the Indel filter expression was set as $\mathrm{QD}<2.0 \|$ ReadPosRankSum $<-10.0$ || InbreedingCoeff $<-0.8 \|$ FS $>100.0 \|$ SOR $>5.0 \|$ QUAL $<30$. Only insertions and deletions shorter than or equal to 40 bp were considered. Indels and SNPs with none bi-allelic, $>40 \%$ missing calls and $\mathrm{MAF}<0.005$ were removed, which yielded the basic set. SNPs with MAF $<0.05$ were further removed for phylogenetic tree structure, IBD calculation, LD decay, PCA and population structure analyses (the core set).

Copy number variations (CNVs) were detected using CNVcaller ${ }^{55}$. Briefly, the reference genome was segmented into overlapping $800 \mathrm{bp}$ sliding windows, and the windows were indexed to form a reference database used in all samples. Then, the reads count of each window across genome from BAM file and a comparable read depth (RD) file of each individual was calculated. The normalized RD files of all samples were piled up into a two-dimensional population RD file, and the integrated $\mathrm{CNV}$ regions (CNVR) were detected by scanning the population $\mathrm{RD}$ file with aberrant $\mathrm{RD}, \mathrm{CNV}$ allele frequency, and significant correlation with adjacent windows. The adjacent candidate windows showing high correlation were further merged.

SNPs and Indels annotation were performed according to the grapevine genome using the package ANNOVAR (Version: 2015-12-14) 56 . The coverage of each accession against each chromosome of grapevine genome was counted base on aligned BAM file using SAMtools (Version: 1.3.1) ${ }^{53}$ software. SNP density, indel density, and total genetic diversity across each chromosome were counted with $100 \mathrm{~kb}$ sliding window using VCFtools software (v0.1.13) ${ }^{57}$.

Population genetics analysis. We used the whole-genome SNPs to construct the ML phylogenetic tree with 100 bootstrap using SNPhylo ${ }^{58}$ (Version: 20140701). Parthenocissus tricuspidata (Boston ivy) was used to provide outgroup information at corresponding positions. The tool iTOL (http://itol.embl de) was used to color the phylogenetic tree. For each group based on the phylogenetic tree, the uncertain samples were discarded in further analyses unless specified otherwise.

SNPs in LD were filtered using PLINK (Version v1.90b3.38) ${ }^{59}$ with a window size of 50 SNPs (advancing 5 SNPs at a time) and an $r^{2}$ threshold of 0.5. PCA was performed with the Genome-wide Complex Trait Analysis (GCTA, version: 1.25.3) software $^{60}$, and the first three eigenvectors were plotted. Population structure was analyzed using the ADMIXTURE (Version: 1.3$)^{61}$ program with a block-relaxation algorithm. To explore the convergence of individuals, we predefined the number of genetic clusters $K$ from 2 to 8 and ran the cross-validation error $(\mathrm{CV})$ procedure. Default methods and settings were used in the analyses.

LD was calculated using PopLDdecay (Version: v3.31, https://github.com/BGIshenzhen/PopLDdecay). The pairwise $r^{2}$ values within and between different chromosomes were calculated. The LD for each group was calculated using SNP pairs only from the corresponding group.

Estimation of mutation rate in grapevines. Since previous research work ${ }^{6}$ used a mutation rate derived from Brassicaceae plants $\left(\mu=2.5 \times 10^{-9}\right)$, we decided to estimate the mutation rate of grapevine. OrthoMCL ${ }^{62}$ were used for defining 
single-copy orthologous genes from five species (V. vinifera, Prunus persica, Arabidopsis thaliana, Theobroma cacao, and Populus trichocarpa, from Ensembl Plant Release 31). Multiple single-copy genes were aligned using Muscle ${ }^{63}$. The four-fold degenerate sites were extracted from each gene and concatenated into a supergene for each species to feed to MrBayes (http://mrbayes.sourceforge.net) to infer the species phylogeny using a ML approach. To estimate the divergence time of each species, the information about the fossil-calibrated divergence time between these species was collected from TimeTree (http://www.timetree.org/). The topology of the ML tree was fed to MCMCTREE in paml version $4.4^{64}$ for constructing a divergence time tree and calculating the divergence time (Supplementary Fig. 13). As a result, the divergence between $V$. vinifera and $P$. persica was estimated to have occurred 104 million years ago. We then identified syntenic regions between the $V$. vinifera and P. persica using LASTZ (http://www.bx.psu.edu/miller_lab/) with $\mathrm{T}=2, \mathrm{C}=2, \mathrm{H}=2000, \mathrm{Y}=3400, \mathrm{~L}=6000$, and $\mathrm{K}=2200$. The polymorphic loci were determined according to the following standards: (1) The nucleotide from either target or query was not classified as a $N$ or $n$; (2) The locus was not in an alignment gap. The sequence divergence between the $V$. vinifera and $P$. persica was estimated to be $37.2 \%$. A mean generation time $(g)$ for grapevine was set at 3 years 6,10 . The final substitution rate per nucleotide per year $(\mu)$ was calculated as $(0.372 \times 3) /\left(2 \times 104 \times 10^{6}\right)=5.4 \times 10^{-9}$ mutations per year for the grapevine. This number is in line with a previous average estimate $\left(5 \times 10^{-9}\right.$ to $\left.7 \times 10^{-9}\right)$ for plant nuclear genes ${ }^{65}$.

Demographic history reconstruction using MSMC. We employed the MSMC 32 model to infer population size $\left(N_{e}\right)$. The input files for MSMC were generated according to MSMC Tools (https://github.com/stschiff/msmc-tools). In brief, only sites with uniquely mapped reads and sites with coverage depths between 0.5 -fold and 2-fold of mean depth were used in the analyses. The remaining genomic regions were masked using the script bamCaller.py. Then all segregating sites within each group were phased using SHAPEIT (Version: v2.r837) ${ }^{66}$, based on a genetic map ${ }^{67}$. Because of the low quality of chromosome 15 , an in-house genetic map of Chr. 15 was used for phasing, which was constructed using Beifeng (maternal parent). A generation time of 3 years and a mutation rate of $5.4 \times 10^{-9}$ mutations per nucleotide per year were used to convert the scaled times and population sizes into real times and sizes. Divergence time between the WEU and CEU population was estimated using MSMC2 (https://github.com/stschiff/ msmc2).

Demographic history inference using SMC ++ . SMC ++ (version: v1.11.1.dev0) ${ }^{33}$ was employed to infer population size histories and split times between the wild European grapevine and domesticated grapevine. According to the phylogenetic tree (Fig. 2a), 60 wild European grapevine accessions and 218 domesticated grapevine accessions (tetraploid excluded) were used for the SMC ++ analysis. For the split analysis, 14 cultivars from the pan-Black Sea, Western European, and East Asian regions were used together with 14 WEU samples. We performed the analysis by masking all selective sweep regions (see below). A generation time of 3 years and a mutation rate of $5.4 \times 10^{-9}$ mutations per nucleotide per year were used to convert the scaled times and population sizes into real times and sizes.

Pedigree construction. We calculated IBD for all pairwise comparisons among the 476 Vitis accessions using PLINK ${ }^{59}$ according to a method published elsewhere ${ }^{10}$. Pairs of accessions are considered to be genetically identical, if they had an IBD > $95 \%$. We used the distribution pattern of all pairwise IBD values (Supplementary Fig. 6a) to determine the cut-off value for first-degree relatives (IBD value $\geq 0.42$ ). We also used the lowest pairwise IBD value $(\geq 0.466)$ from 43 confirmed parentoffspring relationships mentioned in a previous study as a more stringent empirical cut-off ${ }^{10}$. The network images base on IBD were generated using Cytoscape (Version 3.6.0, http://www.cytoscape.org/).

Genome scanning for selective sweep signals. SweeD (Version 3.3.1) ${ }^{68}$ was used to detect selective sweeps based on the CLR test to detect signatures of artificial selection and natural selection in WEU and CEU accessions, respectively. We also performed a genetic differentiation $\left(F_{\mathrm{ST}}\right)$ and polymorphism levels $(\theta \pi$, pairwise nucleotide variation as a measure of variability) based cross approach to investigate the selection signals across the whole genome. A $100 \mathrm{~kb}$ sliding window with $10 \mathrm{~kb}$ step approach was applied to quantify $F_{\mathrm{ST}}$ and $\theta \pi$ by using VCFtools software $(\mathrm{v} 0.1 .13)^{57}$. The candidates that meet both top $5 \%$ of the two values were selected as selective signals.

The specific mutation favored by selection in selective sweeps (identified by the $\theta \pi$ and $F_{\mathrm{ST}}$ cross approach) was captured with phased genotypes using iSAFE $(\mathrm{v} 1.0)^{41}$ with parameter --window 36 --step 18 --MaxRank 2 --MaxFreq 0.9.

Grapevine traits collection. The panicle type was collected by direct observation and judgement. About 50 ripe berries were collected from each available Vitis accession. After berry weight and berry shape were recorded, the samples were crushed in a hand juicer where the number of seeds was counted. The must was collected in a $50 \mathrm{ml}$ centrifuge tube and centrifuged at $5000 \mathrm{~g}$ for $6 \mathrm{~min}$. The supernatant was snap frozen in liquid nitrogen and stored at $40^{\circ} \mathrm{C}$ for later analysis of sugars and acid contents. $1 \mathrm{ml}$ of each sample was passed through a Supelclean ${ }^{T M}$ ENVI LC-18 SPE cartridge (Sigma-Aldrich, St. Louis, USA), and then diluted to $5 \mathrm{ml}$ with distilled water. The diluted sample was passed through a $0.22 \mu \mathrm{m}$ membrane filter. The sugar and acid contents were analyzed using a Dionex P680 HPLC system. The sugar contents were detected using a Shodex RI 101 refractive index detector with reference cell maintained at $40^{\circ} \mathrm{C}$. The SugarPak I column $(300 \mathrm{~mm} \times 6.5 \mathrm{~mm}$ I.D., $10 \mu \mathrm{m}$ particle size, Waters, USA $)$ with a Sugar-Pak I Guard-PakInsert ( $10 \mu \mathrm{m}$ particle size, Waters, USA) was used. The column was maintained at $90^{\circ} \mathrm{C}$ with a Dionex TCC-100 thermostat column compartment. Samples were eluted with double-distilled water. The flow rate was $0.6 \mathrm{ml} / \mathrm{min}$. The Chromeleon chromatography data system was used to integrate peak areas according to external standard solution (Sigma-Aldrich, St. Louis, USA). Malic and tartaric acid contents were detected using a Dionex PDA-100 detector. The Dikma PLATISIL ODS column $(250 \mathrm{~mm} \times 4.6 \mathrm{~mm} \mathrm{I}$. D., $5 \mu \mathrm{m}$ particle size, Dikma, China) with a DikmaSpursil C18 Guard Cartridge $(3 \mu \mathrm{m}, 10 \mathrm{~mm} \times 2.1 \mathrm{~mm}$, Dikma, China) was used. The column was maintained at $40^{\circ} \mathrm{C}$. Samples were eluted with $0.02 \mathrm{~mol} / \mathrm{L} \mathrm{KH}_{2} \mathrm{PO}_{4}$ solution at $\mathrm{pH} 2.4$. The flow rate was $0.8 \mathrm{ml} / \mathrm{min}$. Eluted compounds were detected by UV absorbance at $210 \mathrm{~nm}$. Acid concentration was determined according to external standard solution calibrations.

A new batch of grape berry samples were snap frozen in liquid nitrogen and stored at $-40{ }^{\circ} \mathrm{C}$. The frozen berries were crushed with a mortar and a pestle to remove seeds. The flesh and skin tissues were ground in an IKA A11 mill (IKA Works Inc., Germany) while frozen. A puree was prepared by pulverizing $50 \mathrm{~g}$ pitted frozen berries with $5 \mathrm{~g} \mathrm{CaCl}_{2}$ to decrease the rate of enzymatic reactions. A $1 \mathrm{~cm}$ SPME fiber coated with 50/30 $\mu \mathrm{m}$ divinylbenzene/carboxen/ polydimethylsiloxane (Supelco Inc., Bellefonte, PA) was used to conduct the headspace solid-phase microextraction. Five grams of the puree was placed in a $20 \mathrm{ml}$ capped vial with $10 \mu \mathrm{l}$ of $32.84 \mathrm{mg} / \mathrm{L}$ 3-octanol/ethanol solution, which was used as an internal standard for quantification. The samples were stirred at $40^{\circ} \mathrm{C}$ and after $20 \mathrm{~min}$ of equilibration between the solution and the headspace, the fiber was exposed to the headspace of the capped vial for a period of $30 \mathrm{~min}$. The fiber was then withdrawn and introduced into the injection port of the GC for desorption at $250^{\circ} \mathrm{C}$ for $4 \mathrm{~min}$ in the splitless mode.

GC-MS analysis. Qualitative analysis of the volatile compounds was performed according to a modified method described elsewhere ${ }^{69}$. In brief, the analysis was performed using an Agilent 7890 gas chromatograph equipped with a DB17MS capillary column $(30 \mathrm{~m} \times 0.25 \mathrm{~mm} \times 0.25 \mu \mathrm{m}$; J\&W, Folsom, CA $)$, coupled to an Agilent 5975C quadrupole mass spectrometer (Agilent, Santa Clara, CA). The oven program was as follows: $40^{\circ} \mathrm{C}$ for $5 \mathrm{~min}, 40-70^{\circ} \mathrm{C}$ with gradual increase of $2{ }^{\circ} \mathrm{C} / \mathrm{min}, 70^{\circ} \mathrm{C}$ for $2 \mathrm{~min}, 70-120^{\circ} \mathrm{C}$ with gradual increase of $3{ }^{\circ} \mathrm{C} / \mathrm{min}$, $120-150^{\circ} \mathrm{C}$ with gradual increase of $5^{\circ} \mathrm{C} / \mathrm{min}, 150-220^{\circ} \mathrm{C}$ at $10^{\circ} \mathrm{C} / \mathrm{min}$, and then $220^{\circ} \mathrm{C}$ for $2 \mathrm{~min}$. The injector temperature was maintained at $250^{\circ} \mathrm{C}$, and the transfer line temperature was $280^{\circ} \mathrm{C}$. The ion source temperature was $230^{\circ} \mathrm{C}$ The electronic impact (EI) was $70 \mathrm{eV}$, scanned in the range of $\mathrm{m} / z 30-300$ at a rate of $2.88 \mathrm{scans} / \mathrm{s}$. Helium was employed as a carrier gas, and was introduced at a flow rate of $1 \mathrm{ml} / \mathrm{min}$. A tentative identification of volatile compounds present was achieved by comparing the observed mass spectra with the data system library (NIST2008) and published spectra (Mass Spectrometry Data Center, 1974), supported by retention index data, which were then compared against available literature listing known retention indices (NIST Chemistry WebBook, 2005). All compounds were quantified as 3-octanol equivalents.

Genome-wide association analysis. To minimize false positives and increase statistical power, population structure and cryptic relationships were considered. A compressed mixed linear model program, GAPIT (Version: 2016.03.01) $)^{70}$ was used for the association analysis. The chromosome SNPs were further filtered by a maximum missing rate greater than $30 \%$ and $\mathrm{MAF}<0.05$, and the cluster SNPs (3 SNPs exit within $10 \mathrm{bp}$ ) were also removed. The first three PCA values (eigenvectors), which were derived from wholegenome SNPs, were used as fixed effects in the mixed model to correct for stratification $^{71}$.

We defined the whole-genome significance cutoff with the adjusted Bonferroni test threshold, which was set as $P<0.05 /$ total SNPs. For aroma traits, there are 8,734,701 SNPs from 185 accessions, therefore $\log _{10}(P)=-8.24$. For SUC, TAR, MAL, TA, and Panicle type traits, there are 9,068,232 SNPs from 222 accessions, therefore $\log _{10}(P)=-8.26$. For Color, Weight, Shape, Brix, Seed traits, there are 9,191,395 SNPs from 334 accessions, therefore $\log _{10}$ $(P)=-8.26$

Reporting summary. Further information on experimental design is available in the Nature Research Reporting Summary linked to this article.

\section{Data availability}

The WGRS data set generated and analyzed in the current study is available from NCBI under the BioProject accession PRJNA393611. Data supporting the findings of this work are available within the paper and its Supplementary Information files. The source data of Fig. 5 and Supplementary Figs. 2 and 6 are provided as a Source Data file. All data are 
available from the corresponding author upon reasonable request. A reporting summary for this article is available as a Supplementary Information file.

Received: 3 May 2018 Accepted: 21 February 2019

Published online: 13 March 2019

\section{References}

1. Emanuelli, F. et al. Genetic diversity and population structure assessed by SSR and SNP markers in a large germplasm collection of grape. BMC Plant Biol. 13, 39 (2013).

2. Janick, J. \& Paull, R. E. The Encyclopedia of Fruit and Nuts (CABI Publishing, Oxford, United Kingdom, 2008).

3. This, P., Lacombe, T. \& Thomas, M. R. Historical origins and genetic diversity of wine grapes. Trends Genet. 22, 511-519 (2006).

4. Myles, S. et al. Rapid genomic characterization of the genus vitis. PLoS ONE 5, e8219 (2010).

5. Laucou, V. et al. Extended diversity analysis of cultivated grapevine Vitis vinifera with 10K genome-wide SNPs. PLoS ONE 13, e0192540 (2018).

6. Zhou, Y., Massonnet, M., Sanjak, J. S., Cantu, D. \& Gaut, B. S. Evolutionary genomics of grape (Vitis vinifera ssp. vinifera) domestication. Proc. Natl Acad. Sci. USA 114, 11715-11720 (2017).

7. Tabidze, V. et al. Whole genome comparative analysis of four Georgian grape cultivars. Mol. Genet. Genomics 292, 1377-1389 (2017).

8. Mercenaro, L., Nieddu, G., Porceddu, A., Pezzotti, M. \& Camiolo, S. Sequence polymorphisms and structural variations among four grapevine (Vitis vinifera L.) cultivars representing Sardinian agriculture. Front. Plant Sci. 8, 1279 (2017).

9. McGovern, P. et al. Early Neolithic wine of Georgia in the South Caucasus. Proc. Natl Acad. Sci. USA 114, e10309-e10318 (2017).

10. Myles, S. et al. Genetic structure and domestication history of the grape. Proc. Natl. Acad. Sci. USA 108, 3530-3535 (2011).

11. Arroyo-Garcia, R. et al. Multiple origins of cultivated grapevine (Vitis vinifera L. ssp. sativa) based on chloroplast DNA polymorphisms. Mol. Ecol. 15, 3707-3714 (2006).

12. Jaillon, O. et al. The grapevine genome sequence suggests ancestral hexaploidization in major angiosperm phyla. Nature 449, 463-467 (2007).

13. Duan, N. et al. Genome re-sequencing reveals the history of apple and supports a two-stage model for fruit enlargement. Nat. Commun. 8, 249 (2017).

14. Wang, X. et al. Genomic analyses of primitive, wild and cultivated citrus provide insights into asexual reproduction. Nat. Genet. 49, 765-772 (2017).

15. Varshney, R. K. et al. Whole-genome resequencing of 292 pigeonpea accessions identifies genomic regions associated with domestication and agronomic traits. Nat. Genet. 49, 1082-1088 (2017).

16. Aflitos, S. et al. Exploring genetic variation in the tomato (Solanum section Lycopersicon) clade by whole-genome sequencing. Plant J. 80, 136-148 (2014).

17. Zhou, Z. \& Jiang, Y. Resequencing 302 wild and cultivated accessions identifies genes related to domestication and improvement in soybean. Nat Biotechnol. 33, 408-414 (2015).

18. 3,000 Rice Genomes Project. The 3,000 rice genomes project. Gigascience 3, 7 (2014).

19. Jun Wen et al. Chloroplast phylogenomics of the New World grape species (Vitis, Vitaceae). J. Syst. Evol. 56, 297-308 (2018).

20. Klein, L. L. et al. High-throughput sequencing data clarify evolutionary relationships among North American Vitis species and improve identification in USDA Vitis germplasm collections. Am. J. Bot. 105, 215-226 (2018).

21. Wan, Y. et al. A phylogenetic analysis of the grape genus (Vitis L.) reveals broad reticulation and concurrent diversification during neogene and quaternary climate change. BMC Evol. Biol. 13, 141 (2013).

22. Trondle, D. et al. Molecular phylogeny of the genus Vitis (Vitaceae) based on plastid markers. Am. J. Bot. 97, 1168-1178 (2010).

23. Remington, D. L. et al. Structure of linkage disequilibrium and phenotypic associations in the maize genome. Proc. Natl Acad. Sci. USA 98, 11479-11484 (2001).

24. Marrano, A., Micheletti, D., Lorenzi, S., Neale, D. \& Grando, M. S. Genomic signatures of different adaptations to environmental stimuli between wild and cultivated Vitis vinifera L. Hortic. Res. 5, 34 (2018).

25. Nicolas, S. D. et al. Genetic diversity, linkage disequilibrium and power of a large grapevine (Vitis vinifera $\mathrm{L}$ ) diversity panel newly designed for association studies. BMC Plant Biol. 16, 74 (2016).

26. Huang, $X$. et al. A map of rice genome variation reveals the origin of cultivated rice. Nature 490, 497-501 (2012).
27. Hufford, M. B. et al. Comparative population genomics of maize domestication and improvement. Nat. Genet. 44, 808-811 (2012).

28. Beissinger, T. M. et al. Recent demography drives changes in linked selection across the maize genome. Nat. Plants 2, 16084 (2016).

29. Wang, L. et al. The interplay of demography and selection during maize domestication and expansion. Genome Biol. 18, 215 (2017).

30. Meyer, R. S. et al. Domestication history and geographical adaptation inferred from a SNP map of African rice. Nat. Genet. 48, 1083-1088 (2016).

31. Cubry, P. et al. The rise and fall of African rice cultivation revealed by analysis of 246 new genomes. Curr. Biol. 28, 2274-2282 (2018).

32. Schiffels, S. \& Durbin, R. Inferring human population size and separation history from multiple genome sequences. Nat. Genet. 46, 919-925 (2014).

33. Terhorst, J., Kamm, J. A. \& Song, Y. S. Robust and scalable inference of population history from hundreds of unphased whole genomes. Nat. Genet. 49, 303-309 (2017).

34. De Andres, M. T. et al. Genetic diversity of wild grapevine populations in Spain and their genetic relationships with cultivated grapevines. Mol. Ecol. 21 800-816 (2012)

35. Riaz, S. et al. Genetic diversity analysis of cultivated and wild grapevine (Vitis vinifera L.) accessions around the Mediterranean basin and Central Asia. BMC Plant Biol. 18, 137 (2018).

36. Fechter, I. et al. Candidate genes within a $143 \mathrm{~kb}$ region of the flower sex locus in Vitis. Mol. Genet. Genomics 287, 247-259 (2012).

37. Picq, S. et al. A small XY chromosomal region explains sex determination in wild dioecious $V$. vinifera and the reversal to hermaphroditism in domesticated grapevines. BMC Plant Biol. 14, 229 (2014).

38. Repka, V., Čarná, M. \& Pavlovkin, J. Methyl jasmonate-induced cell death in grapevine requires both lipoxygenase activity and functional octadecanoid biosynthetic pathway. Biologia 68, 896-903 (2013).

39. Gomez, C. et al. In vivo grapevine anthocyanin transport involves vesiclemediated trafficking and the contribution of anthoMATE transporters and GST. Plant J. 67, 960-970 (2011).

40. Kuhn, N. et al. Berry ripening: recently heard through the grapevine. J. Exp. Bot. 65, 4543-4559 (2014)

41. Akbari, A. et al. Identifying the favored mutation in a positive selective sweep Nat. Methods 15, 279-282 (2018).

42. Yakushiji, H. et al. A skin color mutation of grapevine, from black-skinned Pinot Noir to white-skinned Pinot Blanc, is caused by deletion of the functional VvmybA1 allele. Biosci. Biotechnol. Biochem. 70, 1506-1508 (2006).

43. Migicovsky, Z. et al. Patterns of genomic and phenomic diversity in wine and table grapes. Hortic. Res. 4, 17035 (2017).

44. Jenrich, R. et al. Evolution of heteromeric nitrilase complexes in Poaceae with new functions in nitrile metabolism. Proc. Natl Acad. Sci. USA 104, 18848-18853 (2007).

45. Hardie, D. G. Plant protein serine/threonine kinases: classification and functions. Annu. Rev. Plant Physiol. Plant Mol. Biol. 50, 97-131 (1999).

46. Wang, W. et al. Genomic variation in 3,010 diverse accessions of Asian cultivated rice. Nature 557, 43-49 (2018).

47. Grassi F., De Mattia F., Zecca G., Sala F., \& Labra M. Historical isolation and quaternary range expansion of divergent lineages in wild grapevine. Biol. J. Linn. Soc. 95, 611-619 (2008).

48. Nick, P. Schützen und nützen-von der erhaltung zur anwendung. Fallbeispiel europäische wildrebe. In Handbuch Genbank WEL: HOPPEA Denkschriften der Regensburgischen Botanischen Gesellschaft Sonderband (Verlag der gesellschaft, Regensburg, 2014).

49. Hewitt, G. The genetic legacy of the Quaternary ice ages. Nature 405, 907-913 (2000).

50. deMenocal, P. B. Cultural responses to climate change during the late Holocene. Science 292, 667-673 (2001).

51. Patel, R. K. \& Jain, M. NGS QC Toolkit: a toolkit for quality control of next generation sequencing data. PLoS ONE 7, e30619 (2012).

52. Li, H. \& Durbin, R. Fast and accurate short read alignment with Burrows-Wheeler transform. Bioinformatics 25, 1754-1760 (2009).

53. $\mathrm{Li}, \mathrm{H}$. et al. The sequence alignment/map format and SAMtools. Bioinformatics 25, 2078-2079 (2009).

54. McKenna, A. et al. The Genome Analysis Toolkit: a MapReduce framework for analyzing next-generation DNA sequencing data. Genome Res. 20, 1297-1303 (2010)

55. Wang, X. et al. CNVcaller: highly efficient and widely applicable software for detecting copy number variations in large populations. Gigascience 6, 1-12 (2017).

56. Wang, K., Li, M. \& Hakonarson, H. ANNOVAR: functional annotation of genetic variants from high-throughput sequencing data. Nucleic Acids Res. 38, e164 (2010).

57. Danecek, P. et al. The variant call format and VCFtools. Bioinformatics 27, 2156-2158 (2011) 
58. Lee, T. H., Guo, H., Wang, X., Kim, C. \& Paterson, A. H. SNPhylo: a pipeline to construct a phylogenetic tree from huge SNP data. BMC Genomics 15, 162 (2014).

59. Purcell, S. et al. PLINK: a tool set for whole-genome association and population-based linkage analyses. Am. J. Hum. Genet. 81, 559-575 (2007).

60. Yang, J., Lee, S. H., Goddard, M. E. \& Visscher, P. M. GCTA: a tool for genome-wide complex trait analysis. Am. J. Hum. Genet. 88, 76-82 (2011).

61. Alexander, D. H., Novembre, J. \& Lange, K. Fast model-based estimation of ancestry in unrelated individuals. Genome Res. 19, 1655-1664 (2009).

62. Li, L., Stoeckert, C. J. Jr. \& Roos, D. S. OrthoMCL: identification of ortholog groups for eukaryotic genomes. Genome Res. 13, 2178-2189 (2003).

63. Edgar, R. C. MUSCLE: multiple sequence alignment with high accuracy and high throughput. Nucleic Acids Res. 32, 1792-1797 (2004).

64. Yang, Z. PAML 4: phylogenetic analysis by maximum likelihood. Mol. Biol. Evol. 24, 1586-1591 (2007).

65. Wolfe, K. H., Sharp, P. M. \& Li, W.-H. Rates of synonymous substitution in plant nuclear genes. J. Mol. Evol. 29, 208-211 (1989).

66. Delaneau, O., Marchini, J. \& Zagury, J. F. A linear complexity phasing method for thousands of genomes. Nat. Methods 9, 179-181 (2011).

67. Yang, S. et al. Next generation mapping of enological traits in an F2 interspecific grapevine hybrid family. PLoS ONE 11, e0149560 (2016).

68. Pavlidis, P., Zivkovic, D., Stamatakis, A. \& Alachiotis, N. SweeD: likelihoodbased detection of selective sweeps in thousands of genomes. Mol. Biol. Evol. 30, 2224-2234 (2013).

69. Yang, C. et al. Volatiles of grape berries evaluated at the germplasm level by headspace-SPME with GC-MS. Food Chem. 114, 1106-1114 (2009).

70. Lipka, A. E. et al. GAPIT: genome association and prediction integrated tool. Bioinformatics 28, 2397-2399 (2012).

71. Price, A. L. et al. Principal components analysis corrects for stratification in genome-wide association studies. Nat. Genet. 38, 904-909 (2006)

\section{Acknowledgements}

The authors thank the National Key Research and Development Program of China (2018YFD1000105), State Key Laboratory of Agricultural Genomics (No. 2011DQ782025), Key Lab of Agricultural Genomics (Chinese Ministry of Agriculture), the Agricultural Breeding Project of Ningxia Hui Autonomous Region (NXNYYZ20150203), and Shenzhen Engineering Laboratory of Crop Molecular Design Breeding of BGI-Shenzhen for sequencing.

\section{Author contributions}

W.C., S.L., Y. Dong, and J. Sheng designed the research. Z.L., S.L., C.L., P.N., Y. Ding, X.L., P.F., and J.J. provided the grapevine samples. J. Shao, F.D, Yifan Zhu, M.Y., Y.L., and X.H. led the genomic DNA preparation. X.N., S.Z., R.M., W.D., H.H., Youyong Zhu, Y. Dong, and J. Sheng led the genome sequencing. Z.L., P.F., Y. Ding, X.L., and J.J. collected phenotype data. Z.L., S.D., Y. Dong, and W.C. analyzed and interpreted the data. S.D., Y. Dong, and W.C. wrote the manuscript.

\section{Additional information}

Supplementary Information accompanies this paper at https://doi.org/10.1038/s41467019-09135-8.

Competing interests: The authors declare no competing interests.

Reprints and permission information is available online at http://npg.nature.com/ reprintsandpermissions/

Journal peer review information: Nature Communications thanks the anonymous reviewers for their contribution to the peer review of this work.

Publisher's note: Springer Nature remains neutral with regard to jurisdictional claims in published maps and institutional affiliations.

(c) (i) Open Access This article is licensed under a Creative Commons Attribution 4.0 International License, which permits use, sharing, adaptation, distribution and reproduction in any medium or format, as long as you give appropriate credit to the original author(s) and the source, provide a link to the Creative Commons license, and indicate if changes were made. The images or other third party material in this article are included in the article's Creative Commons license, unless indicated otherwise in a credit line to the material. If material is not included in the article's Creative Commons license and your intended use is not permitted by statutory regulation or exceeds the permitted use, you will need to obtain permission directly from the copyright holder. To view a copy of this license, visit http://creativecommons.org/ licenses/by/4.0/.

(C) The Author(s) 2019 